\title{
Biography: Wei-Jei Lee, MD, PhD
}

\author{
Kazunori Kasama ${ }^{1}$
}

Published online: 13 September 2021

(C) The Author(s), under exclusive licence to Springer Science+Business Media, LLC, part of Springer Nature 2021

surgery during this period. During the following two decades, his major interest shifted to bariatric surgery.

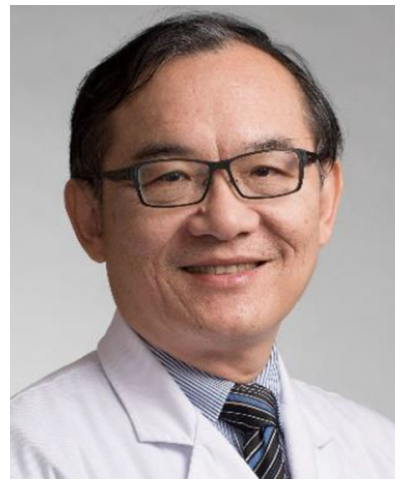

Professor Wei-Jei Lee is a well-known pioneer and researcher in bariatric metabolic surgery (BMS) and is known as the father of Asian BMS. He pioneered the first laparoscopic bariatric surgery in Asia in 1998, and for the past few decades, he has been a huge influence in the careers of many other Asian BMS surgeons.

Professor Lee graduated from the Medical College of National Taiwan University in 1980. He completed his surgical residency training in the National Taiwan University Hospital and became a boarded surgeon in 1986. He finished his $\mathrm{PhD}$ program researching gastric cancer at the Institute of Clinical Medical Research of the National Taiwan University, Medical College in 1994. He became a professor of surgery of the National Taiwan University in 2005.

It has been four decades since Professor Lee first became a physician. During his first decade as a physician, he finished his training in upper gastrointestinal surgery (UGI) and his $\mathrm{PhD}$ program. During the next decade, he focused on gastric cancer surgery and research. He finished a 2-year program in surgical oncology cohosted by the Taiwan Central Research Institute and Harvard University which transformed him into a scientific researcher. He started performing laparoscopic UGI

Kazunori Kasama

k-kasama@mcube.jp

1 Weight Loss and Metabolic Surgery Center, Yotsuya Medical Cube, 7-7 Niban-cho Chiyoda-ku, Tokyo 1020041, Japan
Professor Lee has published more than 300 papers in the field of BMS and was the number one publisher in this field. He has performed many randomized trials in this field and greatly influenced the practice and progress of BMS especially in Asia.

Over the duration of his career, Professor Lee has pioneered many novel BMS in Asia and is especially well-known for being the promoter of the one anastomosis gastric bypass.

Professor Lee is also one of the most important leaders in metabolic surgery for the treatment of type II diabetes mellitus. He organized the Asian Diabetes Study Group and created the ABCD Diabetes Surgery Score. He also strongly advocates for the importance of considering metabolic surgery for early onset diabetes in Asians.

Professor Lee organized the first international bariatric surgery society, the Asian Pacific Metabolic and Bariatric Surgery Society (APMBSS), in the Asia Pacific region and hosted the first meeting in Taipei in 2005. Many young surgeons attended the conference and later became leaders in BMS in their countries (Japan, Korea, India, Hong Kong, Singapore, Malaysia, Thailand, and China).

In 2008, Professor Lee launched the Taiwan Society for Metabolic and Bariatric Surgery (TSMBS). and became the first President. He helped build the IFSO-APC chapter and became its President in 2013. Since 2010, Professor Lee has organized the Asian Diabetes Surgery Society (ADSS) meeting every year. He has many MBS fellows in Taiwan, Asia, and the Arab world. Many of these fellows are now the leading surgeons of this field not only in Taiwan, but also in all over Asia and the Middle East.

At present, Professor Lee spends one-third of his time in China setting up a new BMS center in Su Zhou and hopes to contribute his knowledge and experience to spread BMS in other regions of China. Professor Lee currently spends most of his time on the training and education of young surgeons and hopes to complete his ambitious research project known as the Taiwan Diabesity Study.

Publisher's Note Springer Nature remains neutral with regard to jurisdictional claims in published maps and institutional affiliations. 